\title{
Thrombotic thrombocytopenic purpura following salvage chemotherapy with paclitaxel, ifosfamide and cisplatin in a patient with a refractory germ cell tumor: A case report and review of the literature
}

\author{
ARIFE ULAS ${ }^{1}$, KAMILE SILAY $^{2}$, SEMA AKINCI $^{3}$, MUHAMMED BULENT AKINCI $^{4}$, MEHMET ALI SENDUR $^{4}$, \\ DIDEM SENER DEDE ${ }^{4}$, YUNUS HALIL POLAT ${ }^{5}$ and BULENT YALCIN $^{4}$ \\ ${ }^{1}$ Department of Medical Oncology, Ankara Ataturk Training and Research Hospital; \\ ${ }^{2}$ Department of Geriatrics, Faculty of Medicine, Yildirim Beyazit University; \\ ${ }^{3}$ Department of Hematology, Ankara Ataturk Training and Research Hospital; \\ Departments of ${ }^{4}$ Medical Oncology and ${ }^{5}$ Internal Medicine, Yildirim Beyazit University, Ankara, Turkey
}

Received September 8, 2014; Accepted April 29, 2015

DOI: $10.3892 / 01.2015 .3338$

\begin{abstract}
Thrombotic thrombocy topenic purpura (TTP) is a rare form of thrombotic microangiopathy that is characterized by microvascular thrombosis, thrombocytopenia, hemolysis and end organ damage. An extensive variety of drugs, including certain chemotherapeutic agents, have been associated with TTP. However, paclitaxel, cisplatin and ifosfamide regimen (TIP)-induced TTP has not previously been described. The present study reports the case of a 43-year-old patient with a refractory testicular germ cell tumor who developed acute TTP during TIP chemotherapy. Following the third cycle of TIP chemotherapy, the patient developed fever, anemia, thrombocytopenia and confusion. A diagnosis of TTP was established. Plasmapheresis was initiated as daily treatment in the first week, then continued every other day for 4 weeks. TIP chemotherapy was discontinued. The patient's clinical and neurological symptoms improved markedly after a week. Renal function and hemolysis improved, and the patient was discharged in a stable condition. The patient did not develop any complications and has been in remission for 5 months. The Naranjo adverse drug reaction probability scale indicated a likely association between TTP and the TIP chemotherapy regimen in this patient. This case is also investigated with regard to the associated literature to increase the awareness of TTP following chemotherapy.
\end{abstract}

Correspondence to: Dr Arife Ulas, Department of Medical Oncology, Ankara Ataturk Training and Research Hospital, 1 Lodlumlu Street, Bilkent/Çankaya, Ankara 06200, Turkey

E-mail: drarifeulas@hotmail.com

Key words: thrombotic thrombocytopenic purpura, thrombotic microangiopathy, paclitaxel, cisplatin, ifosphamide

\section{Introduction}

Moschowitz initially described thrombotic thrombocytopenic purpura (TTP) in 1925, characterized by hyaline thrombosis in a variety of tissues (1). The classic pentad of TTP characteristics, including microangiopathic hemolytic anemia (MAHA), thrombocytopenia, fever, neurological findings and kidney function abnormalities, was described by Amorosi and Ultmann following a review of 16 cases in 1966 (2).

TTP is a rare but emergent disease associated with increased mortality (3). The estimated incidence of TTP is 3.7 cases per million (3), and the mortality rate has been reported to range from $10-20 \%$ (4). The condition may be either inherited or acquired. The most common form of TTP is the acquired idiopathic form, characterized by an acute attack. Hemolytic uremic syndrome and TTP, which have similar clinicopathological features, are categorized as thrombotic microangiopathic diseases (5).

It is essential to determine the underlying pathology of TTP in order to form a diagnosis and successfully manage the disease. This life-threatening disease may occur secondary to sepsis and malignancy in oncology cases (most likely stomach, colon and breast cancer, and adenocarcinoma cases) (6). An extensive variety of drugs, including certain chemotherapeutic agents, have been associated with TTP $(7,8)$. Chemotherapy-associated TTP and thrombotic microangiopathy (TMA) are observed particularly with the use of mitomycin $\mathrm{C}$, gemcitabine, cisplatin, bleomycin and oxaliplatin (9-14). To the best of our knowledge, the present study is the first reported case of TTP in association with the paclitaxel, cisplatin and ifosfamide regimen (TIP). The study presents the case of a 43-year-old male with a refractory germ cell tumor who developed TTP following the third cycle of TIP. The aim of this study was to review the literature and emphasize the fact that TTP may be observed as an adverse event to chemotherapeutic agents such as TIP. Clinicians may 
Table I. Serial blood test results following chemotherapy.

\begin{tabular}{lccrrr}
\hline Component & Day 1 & Day 2 & Day 4 & Day 6 & Day 10 \\
\hline Hemoglobin, g/dl & 11 & 8.9 & 7.7 & 5.0 & 6 \\
Platelets, $\mathrm{x} 10^{9} / \mathrm{l}$ & 110 & 41 & 6 & 16.0 & 17 \\
Bilirubin, $\mathrm{mg} / \mathrm{dl}$ & 0.4 & 0.8 & 4.3 & 6 & 4 \\
Creatinine, $\mathrm{mg} / \mathrm{dl}$ & 1.5 & 2.6 & 3.4 & 3 & 3 \\
Lactate dehydrogenase, $\mathrm{U} / \mathrm{l}$ & 152 & 191 & 286 & 274 & 220 \\
\hline
\end{tabular}

obtain a dramatic response to TTP if early recognition and management are achieved.

\section{Case report}

A 43-year-old male presented to Ataturk Training and Research Hospital of Yildirim Beyazit University (Ankara, Turkey) with abdominal pain in May 2013. Magnetic resonance imaging showed an invasive mass to the main vessels around left renal artery, between the left kidney and aorta. Laparoscopic biopsy revealed a $8 \times 4 \times 3-\mathrm{cm}$ germ cell tumor, with residual tumor cells at the surgical margin. Left testicular ultrasonography showed a $6 \times 5 \times 2-\mathrm{cm}$ invasive heterogenic mass with calcifications. Following orchiectomy, pathological examination revealed large, clear cytoplasm and dark stained nucleus with increased pleomorphism. The tumor was $>4 \mathrm{~cm}$ in diameter with rete testical invasion. Based on the pathology results, the patient was diagnosed with high-risk classictype seminoma. Four cycles of chemotherapy, consisting of bleomycin [30,000 IU; intravenous (IV); days 1, 8 and 15], etoposide $\left(100 \mathrm{mg} / \mathrm{m}^{2} ; \mathrm{IV}\right.$, days $\left.1-5\right)$ and cisplatin $(20 \mathrm{mg} /$ $\mathrm{m}^{2}$; IV; days 1-5), were consequently administered every three weeks. Post-treatment positron emission tomography/ CT demonstrated a non-homogeneous soft-tissue mass in the left paraaortic area from the level of the left renal hilus to the aortic bifurcation, with moderate enhancement with ${ }^{18} \mathrm{~F}$-fluorodeoxyglucose (maximum standardized uptake vale, 2.43). The patient underwent a retroperitoneal lymph node dissection with retroperitoneal mass and lymph node excision, and the pathology report was again compatible with a seminoma.

A TIP chemotherapy regimen was initiated as salvage treatment following surgery. The regimen consisted of the administration of $250 \mathrm{mg} / \mathrm{m}^{2}$ paclitaxel on day 1 , and $1,500 \mathrm{mg} / \mathrm{m}^{2}$ ifosfamide, $1500 \mathrm{mg} / \mathrm{m}^{2}$ mesna and $25 \mathrm{mg} / \mathrm{m}^{2}$ cisplatin on days $2-5$, every 21 days. Prophylactic granulocyte colony-stimulating factor (480 $\mu \mathrm{g} / \mathrm{day})$ was administered subcutaneously on days 6-12 of each cycle. During the first cycle, despite prophylaxis, the patient developed febrile neutropenia.

Following the third cycle of TIP chemotherapy, the patient developed a fever, anemia and thrombocytopenia, which was considered to be chemotherapy toxicity. The patient also became confused. Serial blood tests revealed progressively worsening thrombocytopenia, anemia, an increased creatinine level (from 1.5 to 3.4), and increased bilirubin, aspartate aminotransferase (AST) and lactate dehydrogenase (LDH) levels (Table I). Peripheral smear evaluation showed fragmented red blood cells and schistocytes. The fluctuations in the mental status of the patient continued and a diagnosis of TTP was established.

Further investigation revealed a negative Coombs test and a normal reticulocyte count, prothrombin time and partial thromboplastin time. The serum analysis for the a disintegrin and metalloproteinase with a thrombospondin type 1 motif, member 13 (ADAMST 13; also known as von Willebrand factor-cleaving protease) level was in the normal range (79\%; reference range, $40-130 \%$ ). Plasmapheresis was initiated as a daily treatment in the first week, then continued every other day for 4 weeks. TIP chemotherapy was discontinued. The patient's clinical and neurological symptoms improved markedly after a week. Renal functions and hemolysis improved, and the patient was discharged in a stable condition. The patient did not develop any complications and has been in remission for 5 months.

Written informed consent for the present case report was obtained from the patient.

\section{Discussion}

TTP is a form of TMA that is characterized by microvascular thrombosis, thrombocytopenia, hemolysis and end organ damage. MAHA and thrombocytopenia are essential for a diagnosis of TTP. Altered mental status and neurological involvement with focal neurological deficit and epilepsy are common (5). The estimated incidence of TTP is 3.7 cases per million individuals, and this is gradually increasing (3).

Infection, drugs, malignancy, chemotherapy, bone marrow transplantation and pregnancy may all be underlying etiological causes of TTP, but the majority of cases are idiopathic $(6,7)$. Drug-induced TTP has been recognized in the last few decades and accounts for $\sim 15 \%$ of TTP cases (8). TTP/hemolytic uremic syndrome (HUS) or TMA cases induced by chemotherapeutic agents have been reported (9-14). The clinicopathological mechanism of chemotherapy-induced TMA is hyothesized to be dose-dependent toxicity. Mitomycin was the first agent to be linked to TMA in the 1980s. It has been suggested that a cumulative dose effect, immune complex formation and accumulation of these deposits in the renal vasculature are responsible for mitomycin-induced TMA $(9,10)$.

In the present case, the symptoms of anemia, thrombocytopenia, acute renal failure, a fever and mild to moderate confusion were compatible with a diagnosis of TTP. To the best of our knowledge, this study reports the first TIP-induced case of TTP. The TIP salvage regimen is a well-defined, effective treatment for relapsed or refractory germ cell tumors. The Naranjo adverse drug reaction (ADR) scale (15) was applied 
in the present study. This scale indicated a likely association between TTP and TIP chemotherapy, with a score of 6 .

The diagnosis of TTP is usually supported by a low ADAMTS13 level (16). Despite a normal level of ADAMTS13, the current case presented with a pentad of TTP characteristics. With regard to the pathogenesis of TTP, giant von Willebrand factor multimers that are secreted from the endothelium cause thrombocyte adhesion and aggregation (8). Familial TTP is observed in patients with congenital ADAMST13 deficiency, which is responsible from the degradation of these multimers. Immunoglobulin G-type autoantibodies against ADAMST13 are responsible for acquired-type TTP. In drug-induced TTP cases, ADAMST13 deficiency may not be observed (17). In TTP patients who have a normal enzyme level, chemotherapeutic agents cause endothelial toxicity leading to activation of a calpain or other proteases, the decrease of the tissue plasminogen activator level and an increase in the inhibitor of plasminogen activator level (18). Clinicians should establish a diagnosis of TTP and start managing the disease without waiting for the ADAMST13 activity level.

It is difficult to understand the cause-reason association between TTP and chemotherapeutic agents, since malignancy itself may cause TTP. In the present case, the clinical presentation of the patient and the timing of the symptoms were suggestive of chemotherapy-induced TTP. The close association between the symptoms and the timing of TIP administration was suggestive for direct chemotherapy-induced endothelial toxicity. On the other hand, the absence of neurological symptoms, acute renal failure and hemolysis during the previous cycles may have been due to a cumulative effect. It has previously been reported that gemcitabine-induced TTP may be dose-related (19). The risk would be increased if the gemcitabine dose was $>20 \mathrm{~g} / \mathrm{m}^{2}$. Gemcitabine may trigger endothelial damage leading to increased nitric oxide and von Willebrand factor secretion, and increased tissue plasminogen activator and plasminogen activator inhibitor levels (11). One previous TTP case was reported after a single dose of gemcitabine (12). Another study reported that TTP developed following five cycles of the folinic acid, 5-fluorouracil and oxaliplatin regimen, with a cumulative oxaliplatin dose of $850 \mathrm{mg} / \mathrm{m}^{2}$ (14).

In patients who receve multiple chemotherapeutics, it is difficult to identify the offending agent. In previous studies, TTP/HUS has been described following the use of chemotherapy regimens that included cisplatin $(13,20)$. Recently, two breast cancer cases that were treated with a docetaxel and trastuzumab combination with/without carboplatin and developed TMA were reported $(21,22)$. In the present case, it is possible that either the cisplatin by itself or in combination with the paclitaxel or ifosfamide may have induced immunological or direct endothelial damage, and caused the TTP. Since the treatment regimen included three different agents, it is hard to assign blame to only one agent.

TTP is a rare disease, and the morbidity and mortality rates would increase significantly if there was a delay in the diagnosis and management. The mortality rate was $>90 \%$ prior to the introduction of plasma-based treatment in the 1970s (2), and even though better results have been achieved with the introduction of the plasma exchange treatment, the mortality rate remains as high as $10-20 \%$ (22). The diagnosis of chemotherapy-induced
TTP is challenging, with complex disease management and resistance to treatment. In atypical cases, the establishment of a diagnosis is particularly challenging. If the symptoms such as severe hemolysis, thrombocytopenia and renal failure are compatible with TTP, treatment must be initiated immediately. In the present case, plasmapheresis was initiated as soon as the diagnosis was established. In the first week, neurological improvement occurred, and at the end of the fifth week, full recovery was observed. The most effective treatment of drug-induced TTP is the cessation of the administration of the offending agent. The clinical and laboratory response to plasma exchange is usually observed in the first 3 weeks in $80-90 \%$ of cases. If there is no response to plasma exchange and steroid treatment, other treatment options, including intravenous immunoglobulin, cyclophosphamide, vincristine and rituximab, should be considered (23). The potential role of anti-cluster of differentiation 20 rituximab has been shown in TTP/HUS treatment. A systematic review revealed that the complete remission rate with rituximab was $98 \%$ in idiopathic TTP (24). Additionally, the use of recombinant human soluble thrombomodulin was found to be successful in the management of gemcitabine-bevacizumab-induced TTP (25).

In conclusion, to the best of our knowledge, this study is the first to report a case of TTP that developed following TIP chemotherapy. The clinical presentation of TTP may be easily overlooked and the symptoms may mimic sepsis or chemotoxicity. Symptoms such as anemia, thrombocytopenia, renal failure and neurological impairment are suggestive of a diagnosis of TTP. The present patient recovered completely as a diagnosis was established without any delay and treatment with plasmapheresis was performed immediately. The application of the Naranjo ADR probability scale yielded a score of 6, indicating a likely ADR caused by TIP chemotherapy.

Clinicians should be aware that TTP may develop as a complication of chemotherapeutic agents, including TIP. An early diagnosis and treatment will decrease the mortality risk significantly.

\section{References}

1. Moschowitz E: An acute febrile pleiochromic anemia with hyaline thrombosis of the terminal arterioles and capillaries: An undescribed disease. Arch Intern Med 36: 89, 1925.

2. Amorosi E and Ultmann J: Thrombotic thrombocytopenic purpura: Report of 16 cases and review of the literature. Medicine 45: 139, 1966

3. Török TJ Holman RC and Chorba TL: Increasing mortality from thrombotic thrombocytopenic purpura in the United States-analysis of national mortality data, 1968-1991. Am J Hematol 50: 84-90, 1995.

4. Rock GA, Shumak KH, Buskard NA, Blanchette VS, Kelton JG, Nair RC and Spasoff RA: Comparison of plasma exchange with plasma infusion in the treatment of thrombotic thrombocytopenic purpura. Canadian Apheresis Study Group. N Engl J Med 325: 393-397, 1991.

5. Ruggenenti P, Noris M and Remuzzi G: Thrombotic microangiopathy, hemolytic uremic syndrome, and thrombotic thrombocytopenic purpura. Kidney Int 60: 831-846, 2001.

6. Gordon LI and Kwaan HC: Thrombotic microangiopathy manifesting as thrombotic thrombocytopenic purpura hemolytic uremic syndrome in the cancer patient. Semin Thromb Hemost 25: 217-221, 1999.

7. Murgo AJ: Cancer- and chemotherapy-associated thrombotic microangiopathy. In: Hemolytic-Uremic Syndrome and Thrombotic Thrombocytopenic Purpura (Kidney Disease S v. 10). Kaplan BS, Trompeter RS and Moake JL (eds). Vol 22. Marcel Dekker Ltd, New York, pp271-295, 1992. 
8. Dlott JS, Danielson CF, Blue-Hnidy DE and McCarthy LJ: Drug-induced thrombotic thrombocytopenic purpura/ haemolytic uremic syndrome: A concise review. Ther Apher Dial 8: 102-111, 2004

9. Cantrell JE Jr, Phillips TM and Schein PS: Carcinoma-associated hemolytic-uremic syndrome: A complication of mitomycin $\mathrm{C}$ chemotherapy. J Clin Oncol 3: 723-734, 1985.

10. El-Ghazal R, Podoltsev N, Marks P, Chu E and Saif MW: Mitomycin-C-induced thrombotic thrombocytopenic purpura/hemolytic uremic syndrome: Cumulative toxicity of an old drug in a new era. Clin Colorectal Cancer 10: 142-145, 2011.

11. Zupancic M, Shah PC and Shah-Khan F: Gemcitabine-associated thrombotic thrombocytopenic purpura. Lancet Oncol 8 : 634-641, 2008.

12. De Smet D, Jochmans K and Neyns B: Development of thrombotic thrombocytopenic purpura after a single dose of gemcitabine Ann Hematol 87: 495-496, 2008.

13. Gardner G, Mesler D and Gitelman HJ: Hemolytic uremic syndrome following cisplatin, bleomycin, and vincristine chemotherapy: A report of a case and a review of the literature. Ren Fail 11: 133-137, 1989.

14. Jiaxin N and Martha PM: Oxaliplatin-induced thrombotic thrombocytopenic purpura: Case report and literature review. J Clin Oncol. 2012; 30: 312-314

15. Naranjo CA, Busto U, Sellers EM, Sandor P, Ruiz I, Roberts EA, Janecek E, Domecq C and Greenblatt DJ: A method for estimating the propability of advers drug reactions. Clin Pharmaco Ther 30: 239-245, 1981

16. Sadler JE: Von Willebrand factor, ADAMST13 and thrombotic thrombocytopenic purpura. Blood 112: 11-18, 2008.
17. Zakarija A and Bennett C: Drug-induced thrombotic microangiopathy. Semin Thromb Hemost 31: 681-690, 2005.

18. Tsai HM: Advances in the pathogenesis, diagnosis, and treatment of thrombotic thrombocytopenic purpura. J Am Soc Nephrol 14: 1072-1081, 2003.

19. Kasi PM: Thrombotic thrombocytopenic purpura and gemcitabine. Case Rep Oncol 4: 143-148, 2011.

20. Gradishar WJ, Vokes EE, Ni K and Panje WR: Chemotherapy-related hemolytic-uremic syndrome after the treatment of head and neck cancer. A case report. Cancer 66: 1914-1918, 1999.

21. Siau K and Varughese M: Thrombotic microangiopathy following docetaxel and trastuzumab chemotherapy: A case report. Med Oncol 27: 1057-1059, 2010.

22. Iams W, Beckermann KE, Neff AT, Mayer IA and Abramson VG: Thrombotic microangiopathy during docetaxel, trastuzumab, and carboplatin chemotherapy for early stage HER $2^{+}$breast cancer. A case report. Med Oncol 30: 568, 2013.

23. Pisoni R, Ruggenenti P and Remuzzi G: Drug-induced thrombotic microangiopathy: Incidence, prevention and management. Drug Saf 24: 491-501, 2001

24. Tun NM and Villani GM: Efficacy of rituximab in acute refractory or chronic relapsing non familial idiopathic thrombotic thrombocytopenic purpura: A systematic review with pooled data analysis. J Thromb Thrombolysis 34 : 347-359, 2012.

25. Nishijima Y, Hirata H, Himeno A, et al: Drug-induced thrombotic thrombocytopenic purpura successfully treated with recombinant human soluble thrombomodulin. Intern Med 52: $1111-1114,2013$ 